UT-02-33

\title{
Path Integral for Separable Hamiltonians of Liouville-type
}

\author{
Kazuo Fujikawa \\ Department of Physics, University of Tokyo \\ Bunkyo-ku,Tokyo 113, Japan
}

\begin{abstract}
A general path integral analysis of the separable Hamiltonian of Liouville-type is reviewed. The basic dynamical principle used is the Jacobi's principle of least action for given energy which is reparametrization invariant, and thus the gauge freedom naturally appears. The choice of gauge in path integral corresponds to the separation of variables in operator formalism. The gauge independence and the operator ordering are closely related. The path integral in this formulation sums over orbits in space instead of space-time. An exact path integral of the Green's function for the hydrogen atom in parabolic coordinates is ilustrated as an example, which is also interpreted as one-dimensional quantum gravity with a quantized cosmological constant.
\end{abstract}




\section{Introduction}

In 1979, Duru and Kleinert[1] showed an elegant path integral method to evaluate the Green's function for the hydrogen atom exactly. Two basic ingredients in their method are the use of a re-scaled time variable and the so called Kustaanheimo-Stiefel transformation[2] which reveals the $\mathrm{O}(4)$ symmetry explicitly in the coordinate space. The physical meaning of the "re-scaled time variable" however remained somewhat unclear[3]. I have studied this issue by recognizing the procedure in Ref.[1] as a special case of the general treatment of classically separable Hamiltonian of Liouville-type. The basic dynamical principle involved is then identified as the Jacobi's principle of least action for given energy[4].

The path integral on the basis of the Jacobi's principle of least action is basically static and analogous to geometrical optics, and one deals with a sum over orbits in space instead of space-time. Another characteristics of the Jacobi's principle of least action is that it is reparametrization invariant. The general technique of gauge theory is thus applicable to the evaluation of path integral, and a suitable choice of gauge simplifies the problem such as the hydrogen atom. A simple trick in parabolic coordinates, which was used before in a different context by Ravndal and Toyoda[5], renders the hydrogen atom Hamitonian a separable form of Liouville-type.

The hydrogen atom path integral is also understood as a one-dimensional quantum gravity; an interesting aspect of this picture is that the cosmological constant is quantized[6].

\section{Separable Hamiltonian of Liouville-type}

We start with a separable Hamiltonian

$$
H=\frac{1}{V_{1}\left(q_{1}\right)+V_{2}\left(q_{2}\right)}\left\{\frac{1}{2 m}\left(p_{1}^{2}+p_{2}^{2}\right)+U_{1}\left(q_{1}\right)+U_{2}\left(q_{2}\right)\right\}
$$

where the variables change over $\infty>q_{1}, q_{2}>-\infty$. A general Hamiltonian of Liouville-type is given by

$$
H=\frac{1}{V_{1}\left(Q_{1}\right)+V_{2}\left(Q_{2}\right)}\left\{\frac{1}{2 m W_{1}\left(Q_{1}\right)} P_{1}^{2}+\frac{1}{2 m W_{2}\left(Q_{2}\right)} P_{2}^{2}+U_{1}\left(Q_{1}\right)+U_{2}\left(Q_{2}\right)\right\}
$$


but after a canonical transformation

$$
\begin{aligned}
& \frac{1}{\sqrt{W_{1}\left(Q_{1}\right)}} P_{1}=p_{1} \quad, \quad \int_{0}^{Q_{1}} \sqrt{W_{1}(Q)} d Q=q_{1} \\
& \frac{1}{\sqrt{W_{2}\left(Q_{2}\right)}} P_{2}=p_{2} \quad, \quad \int_{0}^{Q_{2}} \sqrt{W_{2}(Q)} d Q=q_{2}
\end{aligned}
$$

and a suitable redefinition of $V$ and $U$, we can write the Hamiltonian in the form of (2.1).

We may then solve the Schroedinger problem

$$
E \psi=\frac{1}{V_{1}\left(q_{1}\right)+V_{2}\left(q_{2}\right)}\left\{\frac{1}{2 m}\left(\hat{p}_{1}^{2}+\hat{p}_{2}^{2}\right)+U_{1}\left(q_{1}\right)+U_{2}\left(q_{2}\right)\right\} \psi
$$

where

$$
\hat{p}_{l}=-i \hbar \frac{\partial}{\partial q_{l}}
$$

for $l=1,2$, and the volume element $d V$, which renders the Hamiltonian $H$ in (2.4) hermitian, is given by

$$
d V=\left(V_{1}\left(q_{1}\right)+V_{2}\left(q_{2}\right)\right) d q_{1} d q_{2}
$$

The classical Hamiltonian (2.1) does not completely specify the operator ordering in (2.4), and the simplest ordering is adopted here. A precise operator ordering needs to be fixed depending on each explicit example.

One may rewrite the above Schroedinger equation (2.4) as

$$
\hat{H}_{T} \psi=0
$$

with a total Hamiltonian defined by a specific gauge condition,

$$
\hat{H}_{T}=\frac{1}{2 m}\left(\hat{p}_{1}^{2}+\hat{p}_{2}^{2}\right)+U_{1}\left(q_{1}\right)+U_{2}\left(q_{2}\right)-E\left(V_{1}\left(q_{1}\right)+V_{2}\left(q_{2}\right)\right)
$$

The meaning of a total Hamiltonian is clarified later. A general procedure to deal with a completely separated operator $\hat{H}_{T}$ is to consider an evolution operator for a parameter $\tau$ defined by

$$
\left\langle q_{1 b}, q_{2 b}\left|e^{-i \hat{H}_{T} \tau / \hbar}\right| q_{1 a}, q_{2 a}\right\rangle=\left\langle q_{1 b}\left|\exp \left[-(i / \hbar)\left(\frac{1}{2 m} \hat{p}_{1}^{2}+U_{1}\left(q_{1}\right)-E V_{1}\left(q_{1}\right)\right) \tau\right]\right| q_{1 a}\right\rangle
$$




$$
\begin{aligned}
& \times\left\langle q_{2 b}\left|\exp \left[-(i / \hbar)\left(\frac{1}{2 m} \hat{p}_{2}^{2}+U_{2}\left(q_{2}\right)-E V_{2}\left(q_{2}\right)\right) \tau\right]\right| q_{2 a}\right\rangle \\
& =\int \mathcal{D} q_{1} \mathcal{D} p_{1} e^{(i / \hbar) \int_{0}^{\tau}\left\{p_{1} \dot{q}_{1}-\left(\frac{1}{2 m} p_{1}^{2}+U_{1}\left(q_{1}\right)-E V_{1}\left(q_{1}\right)\right)\right\} d \tau} \\
& \times \int \mathcal{D} q_{2} \mathcal{D} p_{2} e^{(i / \hbar) \int_{0}^{\tau}\left\{p_{2} \dot{q}_{2}-\left(\frac{1}{2 m} p_{2}^{2}+U_{2}\left(q_{2}\right)-E V_{2}\left(q_{2}\right)\right)\right\} d \tau}(2.9)
\end{aligned}
$$

The parameter $\tau$ is arbitrary, and by integrating over $\tau$ from 0 to $\infty$ one obtains a physically meaningful quantity

$$
\begin{aligned}
& \left\langle q_{1 b}, q_{2 b}\left|\frac{\hbar}{\hat{H}_{T}}\right| q_{1 a}, q_{2 a}\right\rangle_{\text {semi-classical }} \\
= & i \int_{0}^{\infty} d \tau \frac{1}{\sqrt{2 \pi i \hbar\left(\partial q_{1}(\tau) / \partial p_{1}(0)\right)_{q_{1 a}}}} \frac{1}{\sqrt{2 \pi i \hbar\left(\partial q_{2}(\tau) / \partial p_{2}(0)\right)_{q_{2 a}}}} \\
\times & \exp \left\{(i / \hbar) S_{c l}\left(q_{1 b}, q_{1 a}, \tau\right)+(i / \hbar) S_{c l}\left(q_{2 b}, q_{2 a}, \tau\right)\right\}
\end{aligned}
$$

where we wrote the result of a semi-classical approximation for the path integral[7], though in certain cases one may be able to perform an exact path integral in (2.9). The pre-factor in (2.10) is written in terms of classical paths , for example,

$$
q_{1 c l}(\tau)=q_{1}\left(\tau ; q_{1 a}, p_{1}(0)\right)
$$

Namely, the classical paths dictated by the total Hamiltonian $\hat{H}_{T}$ are expressed as functions of the initial positions and momenta. On the other hand, the classical action $S_{c l}$ is expressed as a function of the initial position, final position and elapsed "time" $\tau$ by eliminating $p_{1}(0)$ dependence;for example,

$$
S_{c l}\left(q_{1 b}, q_{1 a}, \tau\right)=\int_{0}^{\tau}\left\{p_{1} \dot{q}_{1}-\left(\frac{1}{2 m} p_{1}^{2}+U_{1}\left(q_{1}\right)-E V_{1}\left(q_{1}\right)\right)\right\}_{c l} d \tau
$$

with $q_{1}(\tau)=q_{1 b}$. If one solves the Hamilton-Jacobi equation in the form

$$
S\left(q_{1 b}, q_{1 a} ; \tau\right)=-A \tau+S\left(q_{1 b}, q_{1 a} ; A\right)
$$

one treats $A$ as a dynamical variable and regards the above equation as a Legendre transformation defined by

$$
\begin{aligned}
& \frac{\partial S\left(q_{1 b}, q_{1 a} ; \tau\right)}{\partial \tau}=-A \\
& \frac{\partial S\left(q_{1 b}, q_{1 a} ; A\right)}{\partial A}=\tau
\end{aligned}
$$


The variable $A$ is then eliminated. This may be regarded as a classical analogue of uncertainty relation; if one specifies $\tau$, the conjugate variable $A$ becomes implicit. It is well-known that the semi-classical approximation (2.10) is exact for a quadratic system.

We next note the relation for the quantity defined in the left-hand side of $(2.10)$

$$
\begin{aligned}
& \left\langle q_{1 b}, q_{2 b}\left|\frac{1}{\hat{H}_{T}}\right| q_{1 a}, q_{2 a}\right\rangle \\
= & \left\langle q_{1 b}, q_{2 b}\left|\frac{1}{\left(\frac{1}{\hat{V}_{1}\left(q_{1}\right)+\hat{V}_{2}\left(q_{2}\right)}\right) \hat{H}_{T}}\right| q_{1 a}, q_{2 a}\right\rangle \frac{1}{V_{1}\left(q_{1 a}\right)+V_{2}\left(q_{2 a}\right)} \\
= & \left\langle q_{1 b}, q_{2 b}\left|\frac{1}{\hat{H}-E}\right| q_{1 a}, q_{2 a}\right\rangle \frac{1}{V_{1}\left(q_{1 a}\right)+V_{2}\left(q_{2 a}\right)} \\
= & \frac{1}{H\left(q_{1 b}, \frac{\hbar}{i} \frac{\partial}{\partial q_{1 b}}, . .\right)-E}\left\{\frac{1}{\sqrt{V_{1}\left(q_{1 b}\right)+V_{2}\left(q_{2 b}\right)}}\left\langle q_{1 b}, q_{2 b} \mid q_{1 a}, q_{2 a}\right\rangle \frac{1}{\sqrt{V_{1}\left(q_{1 a}\right)+V_{2}\left(q_{2 a}\right)}}\right\}
\end{aligned}
$$

by recalling $(\hat{A} \hat{B})^{-1}=\hat{B}^{-1} \hat{A}^{-1}$. The state vectors in these relations are defined for the volume element $d q_{1} d q_{2}$ as

$$
\int d q_{1} d q_{2}\left|q_{1}, q_{2}\right\rangle\left\langle q_{1}, q_{2}\right|=1
$$

Note that the definition of the $\delta$-function in $\left\langle q_{1}^{\prime}, q_{2}^{\prime} \mid q_{1}, q_{2}\right\rangle=\delta\left(q_{1}^{\prime}-q_{1}\right) \delta\left(q_{2}^{\prime}-q_{2}\right)$ depends on the choice of the volume elememt in (2.16) and thus on the choice of $H_{T}$. The last expression in (2.15) is thus correctly defined for the original Hamiltonian $H$ and the original state $\psi$ in (2.4) with the volume element $d V$ in $(2.6)$, since we have the completeness relation from $(2.16)$

$$
\int\left|q_{1}, q_{2}\right\rangle \frac{d V}{V_{1}\left(q_{1}\right)+V_{2}\left(q_{2}\right)}\left\langle q_{1}, q_{2}\right|=1
$$

The left-hand side of (2.15) thus defines the correct Green's function for the original operator $(\hat{H}-E)^{-1}$ by noting the symmetry in $q_{a}$ and $q_{b}$. One can then define the conventional evolution operator by

$$
\begin{aligned}
& \left\langle q_{1 b}, q_{2 b}\left|e^{-i \hat{H}\left(t_{b}-t_{a}\right) / \hbar}\right| q_{1 a}, q_{2 a}\right\rangle_{c o n v} \\
& =\frac{1}{2 \pi i \hbar} \int_{-\infty}^{\infty} d E e^{-i E\left(t_{b}-t_{a}\right) / \hbar}\left\langle q_{1 b}, q_{2 b}\left|\frac{\hbar}{\hat{H}-i \epsilon-E}\right| q_{1 a}, q_{2 a}\right\rangle \frac{1}{V_{1}\left(q_{1 a}\right)+V_{2}(2.18)}
\end{aligned}
$$


where $\epsilon$ is an infinitesimal positive number. The total Hamiltonian changes for a different choice of gauge condition in the Jacobi's principle of least action to be explained below. Consequently, the volume element, which renders $H_{T}$ hermitian, generally depends on the choice of gauge. In the present case, one has the relation

$$
\begin{aligned}
\left\langle q_{1 b}, q_{2 b} \mid q_{1 a}, q_{2 a}\right\rangle_{\text {conv }} & =\left\langle q_{1 b}, q_{2 b} \mid q_{1 a}, q_{2 a}\right\rangle \frac{1}{V_{1}\left(q_{1 a}\right)+V_{2}\left(q_{2 a}\right)} \\
& =\frac{1}{\sqrt{V_{1}\left(q_{1 b}\right)+V_{2}\left(q_{2 b}\right)}}\left\langle q_{1 b}, q_{2 b} \mid q_{1 a}, q_{2 a}\right\rangle \frac{1}{\sqrt{V_{1}\left(q_{1 a}\right)+V_{2}\left(q_{2 a}\right)}}
\end{aligned}
$$

\section{Jacobi's Principle of Least Action}

The meaning of the total Hamiltonian $H_{T}(2.8)$ becomes transparent if one starts with the Jacobi's principle of least action for a given $E$

$$
\begin{aligned}
S & =\int_{0}^{\tau} d \tau L=\int_{0}^{\tau} d \tau \sqrt{2 m\left[E\left(V_{1}\left(q_{1}\right)+V_{2}\left(q_{2}\right)\right)-\left(U_{1}\left(q_{1}\right)+U_{2}\left(q_{2}\right)\right)\right]\left(\dot{q}_{1}^{2}+\dot{q}_{2}^{2}\right)} \\
& =\int \sqrt{2 m\left[E\left(V_{1}\left(q_{1}\right)+V_{2}\left(q_{2}\right)\right)-\left(U_{1}\left(q_{1}\right)+U_{2}\left(q_{2}\right)\right)\right]\left[\left(d q_{1}\right)^{2}+\left(d q_{2}\right)^{2}\right]}
\end{aligned}
$$

which is reparametrization invariant. One then defines the momenta conjugate to coordinates

$$
p_{l}=\frac{\partial L}{\partial \dot{q}_{l}}=\sqrt{2 m\left[E\left(V_{1}\left(q_{1}\right)+V_{2}\left(q_{2}\right)\right)-\left(U_{1}\left(q_{1}\right)+U_{2}\left(q_{2}\right)\right)\right]} \times \frac{\dot{q}_{l}}{\sqrt{\left(\dot{q}_{1}^{2}+\dot{q}_{2}^{2}\right)}}
$$

and obtains a vanishing Hamiltonian, which is a result of reparametrization invariance, and a first class constraint $\phi$ as the generator of reparametrization gauge symmetry,

$$
\begin{aligned}
H & =p_{l} \dot{q}_{l}-L=0 \\
\phi\left(q_{l}, p_{l}\right) & =\frac{1}{V_{1}\left(q_{1}\right)+V_{2}\left(q_{2}\right)}\left\{\frac{1}{2 m}\left(p_{1}^{2}+p_{2}^{2}\right)+U_{1}\left(q_{1}\right)+U_{2}\left(q_{2}\right)\right\}-E \simeq(
\end{aligned}
$$

Following Dirac[8], one may then define a total Hamiltonian

$$
\begin{aligned}
H_{T} & =H+\alpha\left(q_{l}, p_{l}\right) \phi\left(q_{l}, p_{l}\right) \\
& =\alpha\left(q_{l}, p_{l}\right) \phi\left(q_{l}, p_{l}\right) \simeq 0
\end{aligned}
$$


where an arbitrary function $\alpha\left(q_{l}, p_{l}\right)$ specifies a choiceof gauge or a choice of the arbitrary parameter $\tau$ in (3.1), which parametrizes the orbit for a given $E$. The quantum theory is defined by( up to an operator ordering)

$$
i \hbar \frac{\partial}{\partial \tau} \psi=\hat{H}_{T} \psi
$$

with a physical state condition

$$
\hat{\alpha}\left(q_{l}, p_{l}\right) \hat{\phi}\left(q_{l}, p_{l}\right) \psi_{p h y}=0
$$

A choice of the specific gauge $\alpha\left(q_{l}, p_{l}\right)=V_{1}\left(q_{1}\right)+V_{2}\left(q_{2}\right)$ gives rise to (2.7) and the choice $\alpha\left(q_{l}, p_{l}\right)=1$ gives the conventional static Schroedinger equation (2.4), since $\psi$ appearing in these equations are physical states.

The basic dynamical principle involved is thus identified as the Jacobi's principle of least action, which is analogous to geometrical optics, and the formula of an evolution operator (2.9) dictated by (3.5) provides a basis for the path integral approach to a general separable Hamiltonian of Liouvilletype. The path integral in (2.9) deals with a sum over orbits in space instead of space-time, and the notion of re-scaled time does not explicitly appear in the present approach; the evolution operator (2.9) essentially generates a gauge transformation.

\section{Hydrogen Atom}

It is known that the Schroedinger equation for the hydrogen atom is written in parabolic coordinates as

$$
\begin{aligned}
\hat{\tilde{H}}_{T} \psi & =0 \\
\left(\hat{p}_{\varphi}-\hat{p}_{\varphi^{\prime}}\right) \psi & =0
\end{aligned}
$$

where

$$
\begin{aligned}
\hat{\tilde{H}}_{T} & =\frac{1}{2 m}\left[\hat{p}_{u}^{2}+\frac{1}{u^{2}} \hat{p}_{\varphi}^{2}+\hat{p}_{v}^{2}+\frac{1}{v^{2}} \hat{p}_{\varphi^{\prime}}\right]+\frac{m \omega^{2}}{2}\left(u^{2}+v^{2}\right)-e^{2} \\
& =\frac{1}{2 m} \vec{p}_{u}^{2}+\frac{m \omega^{2}}{2} \vec{u}^{2}+\frac{1}{2 m} \vec{p}_{v}^{2}+\frac{m \omega^{2}}{2} \vec{v}^{2}-e^{2}
\end{aligned}
$$


and we defined

$$
\begin{aligned}
\vec{u} & =\left(u_{1}, u_{2}\right)=(u \cos \varphi, u \sin \varphi) \\
\vec{p}_{u}^{2} & =\hat{p}_{u}^{2}+\frac{1}{u^{2}} \hat{p}_{\varphi}^{2} \\
\vec{v} & =\left(v_{1}, v_{2}\right)=\left(v \cos \varphi^{\prime}, v \sin \varphi^{\prime}\right) \\
\vec{p}_{v}^{2} & =\hat{p}_{v}^{2}+\frac{1}{v^{2}} \hat{p}_{\varphi^{\prime}}^{2}
\end{aligned}
$$

The subsidiary condition in (4.1) replaces the use of the Kustaanheimo-Stiefel transformation[2], and at the same time it renders a quadratic Hamiltonian of Liouville-type. This introduction of auxiliary variables (4.3) has been discussed by Ravndal and Toyoda[5].

The general procedure we discussed is thus applicable to the hydrogen atom, and we recover the classical exact result of Duru and Kleinert[1].

\section{Quantum gravity with a quantized cosmological constant}

A alternative way to see the physical meaning of the parameter $\tau$ is to study the one-dimensional quantum gravity coupled to matter variables $\vec{x}$ defined by

$$
\int \frac{\mathcal{D} \vec{x} \mathcal{D} h}{\text { gauge volume }} \exp \left\{\frac{i}{\hbar} \int_{0}^{\tau} L h d \tau\right\}
$$

with

$$
L=\frac{m}{2 h^{2}}\left(\frac{d \vec{x}}{d \tau}\right)^{2}-V(r)+E
$$

where $h$ stands for the einbein, a one-dimensional analogue of vierbein $h_{\mu}^{a}$, and $h=\sqrt{g}$ in one-dimension. If one uses the solution of the equation of motion for $h$ defined by the Lagrangian $\mathcal{L}=L h$, the action in (4.4) is reduced to the one appearing in the Jacobi's principle of least action. An interesting aspect of this interpretation is that one has a toy model of quantum gravity with a quantized cosmological constant[6].

\section{Discussion}

An attempt to solve the Green's function for the hydrogen atom exactly in the path integral[1] opened a new avenue for the path integral treatment of a 
general separable Hamiltonian of Liouville-type. This new view point, which has been shown to be based on the Jacobi's principle of least action , provides a more flexible framework of path integral to deal with a wider class of problems of physical interest. The Jacobi's principle of least action, besides being reparametrization invariant, gives a geometrical picture of particle orbits in a curved space deformed by the potential. On the other hand, the fundamental space-time picture of the conventional Feynman path integral, which is associated with the Hamilton's principle of stationary action, is lost.

I happened to learn the path integral of hydrogen atom through a seminar given by C. Bernido, who provided me references to the works of Kleinert. I wrote two papers on this subject, as quoted in this note[4][6]. Though I have spent much time on the path integral in relativistic quantum field theory, these two papers are the only ones I wrote on the path integral of non-relativistic quantum mechanics. By publishing these two papers, I was embarassed to find an enormous difference of culture between physicists in relativistic and non-relativistic fields of path integral; the major issue in the latter field of non-relativistic path integral is operator ordering, which is not fundamental in relativistic problems mainly due to the strong constraints provided by the symmetry principles such as Lorentz and gauge invariance. An over-emphasis on the operator ordering problem does not appear to be healthy.

I believe that a more fruitful field will open if the researchers in these two realms of path integral work together. Professor H. Kleinert will certainly be one of the leaders in such an attempt.

\section{References}

[1] I. H. Duru and H. Kleinert, Phys. Lett.B84(1979)185.

[2] P. Kustaanheimo and E. Stiefel, J. Reine Angew. Math.218(1965)204.

[3] As for complete references to the related problem, see H. Kleinert, Path Integrals in Quantum Mechanics, Statistics and Polymer Physics(World Scientific, Singapore, 1995).

[4] K.Fujikawa, Nucl. Phys.B484(1997)495.

[5] F. Ravndal and T. Toyoda, Nucl. Phys. B3(1967)312. 
[6] K.Fujikawa, Prog. Theor. Phys. 96(1996)863.

[7] W. Pauli, Ausgewaehlte Kapitel aus der Feldquantisierung, Lecture Notes, Zurich, 1951.

Ph. Choquard, Helv. Phys. Acta 28(1955)89.

[8] P. A. M. Dirac, Lectures on Quantum Field Theory (Yeshiva Univ., New York, 1966). 